\title{
Short telomere length, lung function and chronic obstructive pulmonary disease in 46396 individuals
}

\author{
Line Rode, ${ }^{1}$ Stig E Bojesen, 1,2,3 Maren Weischer, 1,3 Jørgen Vestbo, 4,5 \\ Børge G Nordestgaard ${ }^{1,2,3}$
}

- Additional material is published online only. To view please visit the journal online (http://dx.doi.org/10.1136/ thoraxjnl-2012-202544).

${ }^{1}$ Department of Clinical Biochemistry and the Copenhagen General Population Study, Herlev Hospital, Copenhagen University Hospital, Herlev, Denmark

${ }^{2}$ The Copenhagen City Heart Study, Bispebjerg Hospital, Copenhagen University Hospital, Copenhagen, Denmark

${ }^{3}$ Faculty of Health Sciences, University of Copenhagen, Copenhagen, Denmark

${ }^{4}$ Respiratory Research Group, Manchester Academic Health Sciences Centre, University Hospital South Manchester, NHS Foundation Trust,

Manchester, UK

${ }^{5}$ Department of Respiratory

Diseases, University of Southern Denmark, Odense, Denmark

\section{Correspondence to} Professor Børge $G$ Nordestgaard, Department of Clinical Biochemistry and the Copenhagen General Population Study, Herlev Hospital, Copenhagen University Hospital, Herlev Ringvej 75, Herlev DK-2730, Denmark;

boerge.nordestgaard@regionh. $\mathrm{dk}$

Received 8 August 2012 Revised 28 November 2012 Accepted 29 November 2012 Published Online First 25 December 2012

\author{
ABSTRACT \\ Background A previous case-control study of 100 \\ individuals found that short telomere length was \\ associated with a 28-fold increased risk of chronic \\ obstructive pulmonary disease (COPD).
}

Objectives To test the hypothesis that short telomere length is associated with reduced lung function and an increased risk of COPD.

Methods Observational study of 46396 individuals from the Danish general population.

Measurements Leucocyte telomere length and spirometry were measured. COPD was defined using either fixed forced expiratory volume in $1 \mathrm{~s}\left(\mathrm{FEV}_{1}\right) /$ forced vital capacity (FVC) ratio $<0.70$ as suggested by the Global initiative for chronic Obstructive Lung Disease (GOLD) or $\mathrm{FEV}_{1} / \mathrm{FVC}$ below the lower limit of normal (LLN).

Results Telomere length decreased significantly with increasing age $\left(p<10^{-300}\right)$. FEV 1 , FVC and $\mathrm{FEV}_{1} / \mathrm{FVC}$ decreased with decreasing telomere length quartiles ( $p$ trend: $5 \times 10^{-51}, 5 \times 10^{-35}$ and $6 \times 10^{-137}$, respectively), but the associations attenuated after age and multivariable adjustment. The risk of COPD increased with decreasing telomere length quartile ( $p$ trend: $p=7 \times 10^{-92}$ for GOLD; $\mathrm{p}=8 \times 10^{-44}$ for $\mathrm{FEV}_{1} / \mathrm{FVC}$ below LLN), but associations also attenuated after adjustment. Unadjusted and multivariable adjusted OR for shortest versus longest telomere length quartiles were $2.06(95 \% \mathrm{Cl} 1.91$ to 2.22$)$ and $1.15(95 \% \mathrm{Cl} 1.06$ to 1.25$)$ for GOLD and $1.73(95 \%$ $\mathrm{Cl} 1.60$ to 1.88$)$ and $1.19(95 \% \mathrm{Cl} 1.09$ to 1.30$)$ for $\mathrm{FEV}_{1} /$ FVC below LLN, respectively. Per 1000 base pairs decrease in telomere length, the multivariable adjusted OR was 1.07 (95\% Cl 1.03 to 1.10) for GOLD and 1.07 (95\% Cl 1.03 to 1.11) for $\mathrm{FEV}_{1} / \mathrm{FVC}$ below LLN.

Conclusions Short telomere length is associated with decreased lung function and with increased risk of COPD, but the associations are markedly attenuated after adjustment. Our data support a modest correlation between telomere length and the lung function indices examined.

\section{INTRODUCTION}

Lung tissue is often exposed to oxidants from inhaled nitrogen dioxide, ozone, gasoline and diesel exhaust and from tobacco smoke. The combination of a large surface area and a high blood supply result in a high susceptibility to oxidative stress in lung tissue, ${ }^{1}$ a factor that is believed to play a crucial role in pulmonary inflammation, decreased pulmonary function and in the development of chronic obstructive pulmonary disease (COPD).

\section{Key messages}

What is the key question?

- Are shorter telomeres associated with reduced lung function and increased risk of chronic obstructive pulmonary disease (COPD)?

\section{What is the bottom line?}

- It is biologically plausible that pulmonary oxidative stress and inflammation could lead to short telomere length and, hence, short telomere length could be a marker of decreased lung function and increased risk of COPD.

- Among 46396 individuals, those with short telomeres are more likely to have reduced lung function and COPD, according to the GOLD classification as well as according to forced expiratory volume in $1 \mathrm{~s} /$ forced vital capacity below lower limit of normal, compared with those with long telomeres.

\section{Why read on?}

- This very large study shows that short telomere length is associated with COPD risk. The association may, however, be more moderate than previously suggested in smaller studies of 76-291 individuals.

- Our results stress the importance of adjusting for age and other confounders when examining the association between COPD and short telomeres.

Telomeres are repetitive DNA sequences at the end of chromosomes which are important for chromosome protection and thus for longevity of cells. $^{2}{ }^{3}$ In most cells telomere length shortens with each cell division, and since exposure to oxidative stress as well as inflammation enhance this shortening, telomere length may serve as a marker for cellular ageing. ${ }^{4}$ In accordance with this notion, telomere length decreases with increasing age, and short telomere length is also associated with lifestyle factors such as smoking, obesity and stress. ${ }^{6-9}$ It is therefore plausible that pulmonary oxidative stress and inflammation could lead to short telomere length, and that short telomere length could be a marker of decreased lung function and increased risk of COPD. 
We tested the hypothesis that short telomere length is associated with decreased lung function and increased risk of COPD in a sample of 46396 participants from the Danish general population. Severity of COPD was defined according to forced expiratory volume in $1 \mathrm{~s}\left(\mathrm{FEV}_{1}\right)$ and forced vital capacity (FVC), either by the Global initiative for chronic Obstructive Lung Disease (GOLD) criteria or by $\mathrm{FEV}_{1} / \mathrm{FVC}$ below the lower limit of normal (LLN). ${ }^{10} 11$

\section{METHODS}

\section{Population}

We studied participants from the Copenhagen General Population Study and the Copenhagen City Heart Study, both of which are Danish prospective general population studies. ${ }^{12-14}$ In both studies, randomly selected Copenhagen residents were invited to complete a questionnaire and undergo a physical examination including spirometry. Whole blood samples were collected for DNA isolation. There was no overlap of individuals between the two studies.

The study population consisted of 37355 participants from the Copenhagen General Population Study included between 2003 and 2007 and 9041 participants from the Copenhagen City Heart Study included between 1991 and 1994, all with available spirometry and telomere length measurement. A total of 16563 individuals were invited to participate in The Copenhagen City Heart Study between 1991 and 1994 and of those 10135 entered the study. Whole blood samples for DNA isolation were collected in 9252 individuals, and among these 9041 had information on telomere length and spirometry. The Copenhagen General Population Study is an ongoing study. A total of $46 \%$ of invited individuals participated and $99 \%$ had whole blood samples collected for DNA isolation. Spirometry data are available for $98 \%$ of participants.

\section{Lung function and COPD}

At each examination three sets of values were registered for $\mathrm{FEV}_{1}$ and FVC and the highest set of values was used in the analyses. At least two measurements of $\mathrm{FEV}_{1}$ and FVC with a difference of less than $5 \%$ had to be obtained to ensure correct performance of the procedure. In the Copenhagen General Population Study, two different spirometers were used, a dry wedge spirometer in the first 15000 participants and an EasyOne Spirometer in the rest of the population. In the Copenhagen City Heart Study, a dry wedge spirometer was used. Details regarding instruments have been described elsewhere. $^{12} 1516$

Predicted values for $\mathrm{FEV}_{1}$ and FVC were calculated for never smoking men and women individually using multiple linear regression analysis with age and height as covariates. Regression analyses were performed for the two studies individually as well as for type of spirometer used in the Copenhagen General Population Study. ${ }^{12}{ }^{17}$ LLN for $\mathrm{FEV}_{1} / \mathrm{FVC}$ was defined as a ratio below the 5 th centile of the predicted ratio for height and age in never smoking men and women individually, separately in each study and according to the type of spirometer used.

COPD was defined according to (1) GOLD with $\mathrm{FEV}_{1} /$ $\mathrm{FVC}<0.70$ and degree of airflow limitation split in four grades ${ }^{10}$ : (I) $\mathrm{FEV}_{1} \geq 80 \%$ predicted, (II) $\mathrm{FEV}_{1} 50-79 \%$ predicted, or (III-IV) $\mathrm{FEV}_{1}<50 \%$ predicted; and (2) $\mathrm{FEV}_{1} / \mathrm{FVC}$ below LLN. ${ }^{11}$ Individuals aged $<40$ years with self-reported asthma $(n=323)$ were omitted from all analyses.

\section{Telomere length}

Telomere length was measured in DNA isolated from blood leucocytes. We used a modified monochrome multiplex quantitative PCR method, ${ }^{18}$ as described in detail elsewhere. ${ }^{19}$ In brief, we used the reference single-copy gene for albumin to adjust for different amounts of DNA in samples. DNA from each participant was measured in quadruplicate and the mean of four telomere threshold cycle values and the mean of four albumin threshold cycle values were calculated. Unsuccessful samples were analysed again until valid measurements were available in 99.9\% of participants. Of these, a telomere length measurement was obtained after the first analysis in $98.4 \%$, whereas $1.1 \%$ of samples were rerun once and $0.5 \%$ were rerun twice. The absolute telomere length was derived after calibration with measurement on K562 cell line DNA, included in each plate.

\section{Covariates}

The following information was collected from the questionnaires at inclusion in the studies: age at examination, gender, number of years in school as well as information on smoking habits, occupational exposure to dust and fumes (yes/no) and dyspnoea. Participants were classified as never smokers, former smokers or current smokers. For former smokers and current smokers, packyears of cigarettes or equivalent smoking were calculated and information on inhalation was noted as yes/no. One pack-year corresponds to one pack of 20 cigarettes or equivalent smoked for a year. The severity of dyspnoea was graded from 0 to 4 according to the modified Medical Research Council Dyspnoea Scale. ${ }^{20}$ Questionnaires were completed and reviewed by an examiner on the day of examination. Height and weight were registered on the day of examination. Body mass index was calculated as the measured weight divided by the square of the measured height and categorised according to the WHO classification into body mass index $<18.5 \mathrm{~kg} / \mathrm{m}^{2}, 18.5-24.9 \mathrm{~kg} /$ $\mathrm{m}^{2}, 25-29.9 \mathrm{~kg} / \mathrm{m}^{2}$ and $\geq 30 \mathrm{~kg} / \mathrm{m}^{2}$. The number of years in school were categorised as $<8$ years, $8-11$ years and $>11$ years. For logistic regression analysis, C-reactive protein levels were categorised according to lowest, middle and highest tertiles but used on a continuous scale in other analyses.

\section{Statistical analyses}

The association between telomere length continuously with age and between telomere length quartiles and lung function was assessed by general linear models while the association with COPD was assessed by logistic regression. Multivariable models were adjusted for age, gender, body mass index, smoking status, smoking inhalation, cumulative smoking in pack-years, occupational exposure to dust and fumes, years in school, plasma C-reactive protein levels, grade of dyspnoea and study. Covariates were more than $99.5 \%$ complete; missing values were imputed according to age, gender and smoking status. For trend tests, individuals were categorised according to telomere length quartiles coded 1-4 with the first quartile consisting of individuals with the longest telomere lengths. We tested interaction by using a likelihood ratio test that compared the main effects model with a model also including a two-factor interaction term. Stata V.12.0 was used for all analyses.

\section{RESULTS}

Figure 1 shows the decrease in telomere length on a continuous scale with increasing age in the 46396 participants from the general population $\left(\mathrm{p}<1 \times 10^{-300}\right)$. Decreasing telomere length in quartiles was associated with increased age, male gender, 
Figure 1 Telomere length in base pairs by 5 -year age groups according to age at blood sampling. Based on 46396 participants from the Danish general population from the Copenhagen General Population Study and the Copenhagen City Heart Study.

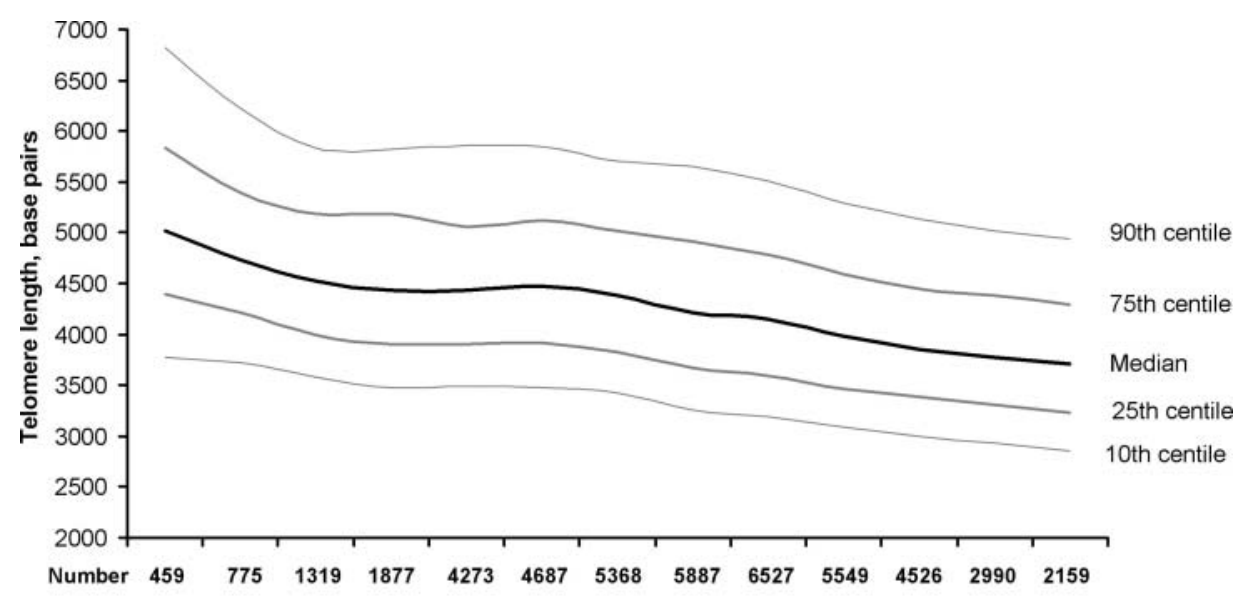

20-24 25-29 $30-34 \quad 35-39 \quad 40-44 \quad 45-49 \quad 50-54 \quad 55-59 \quad 60-64 \quad 65-69$ 70-74 $75-79 \quad 80+$ Age, years increased body mass index, former smoking status, smoking inhalation, increased cumulative smoking, increased occupational exposure to dust and fumes, $<8$ years in school and with increased C-reactive protein levels (table 1). Table 2 shows the association between telomere length on a continuous scale and covariates individually and in combination. The strongest associations with telomere length in the multivariable analysis were found for age, gender, cumulative smoking in pack-years and body mass index.

Decreasing telomere length in quartiles was associated with decreased $\mathrm{FEV}_{1} \%$ predicted ( $\mathrm{p}$ trend: $\mathrm{p}=5 \times 10^{-51}$ ), decreased FVC $\%$ predicted $\left(\mathrm{p}=5 \times 10^{-35}\right)$ and with decreased $\mathrm{FEV}_{1} / \mathrm{FVC}$ $\left(\mathrm{p}=6 \times 10^{-137}\right)$ (figure 2). $\mathrm{FEV}_{1} \%$ predicted was $97 \%$ in the first quartile, $96 \%$ in the second, $95 \%$ in the third and $94 \%$ in the fourth quartile of telomere length. Corresponding values for FVC \% predicted were 99\%, 98\%, 98\% and 97\%, and for $\mathrm{FEV}_{1} / \mathrm{FVC} 0.78,0.780 .77$, and 0.76 , respectively. The association between decreased telomere length and decreased lung function was attenuated after age adjustment ( $\mathrm{p}$ trend: $\mathrm{p}=5 \times 10^{-16}$ for $\mathrm{FEV}_{1} \%$ predicted; $\mathrm{p}=1 \times 10^{-12}$ for $\mathrm{FVC} \%$ predicted; $\mathrm{p}=5 \times 10^{-5}$ for $\mathrm{FEV}_{1} / \mathrm{FVC}$ ) and even further after multivariable adjustment for age, gender, body mass index, smoking status, smoking inhalation, cumulative smoking, occupational exposure to dust and fumes, years in school, C-reactive protein levels, grade of dyspnoea and study ( $\mathrm{p}$ trend: $\mathrm{p}=1 \times 10^{-6}$, $\mathrm{p}=4 \times 10^{-5}$ and $\mathrm{p}=0.07$, respectively).

Similar patterns were seen when analysing telomere length as a continuous variable (ie, change in lung function per 1000 base pair decrease in telomere length; figure 3). Stratified analyses of the change in unadjusted $\mathrm{FEV}_{1} / \mathrm{FVC}$ per 1000 base pair decrease in telomere length showed a decrease in all strata (figure 4). $\mathrm{FEV}_{1} / \mathrm{FVC}$ decreased slightly more in women than in men, in individuals aged $\geq 60$ years than in those aged $<60$ years of age and in former and current smokers than in never smokers.

A total of 6770 participants $(15 \%)$ had an $\mathrm{FEV}_{1} / \mathrm{FVC}<0.70$. Among these, $2922(43 \%)$ were classified as GOLD stage I, $3026(45 \%)$ as GOLD stage II and $822(12 \%)$ as GOLD stages III-IV. A total of 5136 participants (11\%) met the criteria for COPD by $\mathrm{FEV}_{1} / \mathrm{FVC}$ below LLN. Table $\mathrm{S} 1$ in the online supplement shows the baseline characteristics of participants according to these COPD criteria.

Figure 5 shows the unadjusted, age adjusted and multivariable adjusted logistic regression analyses of the association between decreasing telomere length in quartiles and COPD risk according to the GOLD criteria and $\mathrm{FEV}_{1} / \mathrm{FVC}$ below LLN. In the unadjusted analyses, the OR of COPD increased with decreasing telomere length quartiles ( $p$ trend: $p=7 \times 10^{-92}$ for GOLD criteria; $\mathrm{p}=8 \times 10^{-44}$ for $\mathrm{FEV}_{1} / \mathrm{FVC}$ below LLN). In the

Table 1 Baseline characteristics of participants by quartiles of telomere length

\begin{tabular}{|c|c|c|c|c|c|}
\hline & $\begin{array}{l}\text { 1st quartile (longest) } \\
n=11599\end{array}$ & $\begin{array}{l}\text { 2nd quartile } \\
\mathrm{n}=11600\end{array}$ & $\begin{array}{l}\text { 3rd quartile } \\
\mathrm{n}=11599\end{array}$ & $\begin{array}{l}\text { 4th quartile (shortest) } \\
\mathrm{n}=11598\end{array}$ & p For trend \\
\hline Telomere length, base pairs & $5323(5007-5842)$ & $4402(4240-4568)$ & $3821(3684-3956)$ & $3204(2950-3387)$ & \\
\hline Age, years & $53(43-62)$ & $56(46-65)$ & $60(50-69)$ & $65(56-73)$ & $<1 \times 10^{-300}$ \\
\hline Men, $\mathrm{n}(\%)$ & $5026(43)$ & $5155(44)$ & $5433(47)$ & $5740(49)$ & $1 \times 10^{-22}$ \\
\hline Body mass index, $\mathrm{kg} / \mathrm{m}^{2}$ & $25(23-28)$ & $26(23-28)$ & $26(23-29)$ & $26(23-29)$ & $5 \times 10^{-48}$ \\
\hline \multicolumn{6}{|l|}{ Smoking status } \\
\hline Former smoking, $\mathrm{n}(\%)$ & $4236(37)$ & $4512(39)$ & $4787(41)$ & $5121(44)$ & $3 \times 10^{-33}$ \\
\hline Current smoking, $\mathrm{n}(\%)$ & $2857(25)$ & $2758(24)$ & $2909(25)$ & $2841(25)$ & 0.14 \\
\hline Smoking inhalation, $\mathrm{n}$ ( $\%$ of former or current smokers) & $5927(84)$ & $5909(81)$ & $6032(78)$ & $5984(75)$ & $1 \times 10^{-39}$ \\
\hline Cumulative smoking, pack-years & $5(0-22)$ & $6(0-24)$ & $9(0-29)$ & $12(0-32)$ & $4 \times 10^{-103}$ \\
\hline Occupational exposure to dust/fumes, $\mathrm{n}(\%)$ & 1499 (13) & $1531(13)$ & 1650 (14) & $1766(15)$ & $4 \times 10^{-7}$ \\
\hline$<8$ years in school, $n(\%)$ & $1635(14)$ & $1820(16)$ & $2289(20)$ & $2754(24)$ & $4 \times 10^{-94}$ \\
\hline C-reactive protein, $\mathrm{mg} / \mathrm{l}$ & $1.4(0.8-2.6)$ & $1.5(1.0-2.6)$ & $1.6(1.1-2.8)$ & $1.7(1.2-3.1)$ & $5 \times 10^{-152}$ \\
\hline
\end{tabular}


Table 2 Association between telomere length and covariables individually by univariable regression analysis and in combination by multivariable regression analysis

\begin{tabular}{|c|c|c|c|c|c|}
\hline & \multicolumn{2}{|l|}{ Univariable } & \multicolumn{2}{|l|}{ Multivariable } & \multirow[b]{2}{*}{ Rank } \\
\hline & Coefficient $(95 \% \mathrm{Cl})$ & p Value & Coefficient $(95 \% \mathrm{Cl})$ & p Value & \\
\hline Age, years & $-22.1(-22.7$ to -21.4$)$ & $<1 \times 10^{-300}$ & $-21.5(-22.2$ to -20.8$)$ & $<1 \times 10^{-300}$ & 1 \\
\hline Male gender & $-102.1(-120.1$ to -84.1$)$ & $1 \times 10^{-28}$ & $-88.8(-106.0$ to -71.6$)$ & $4 \times 10^{-24}$ & 2 \\
\hline Cumulative smoking in pack-years & $-5.2(-5.6$ to -4.8$)$ & $4 \times 10^{-126}$ & $-1.4(-1.9$ to -0.9$)$ & $3 \times 10^{-8}$ & 3 \\
\hline Body mass index, $\mathrm{kg} / \mathrm{m}^{2}$ & $-13.2(-15.3$ to -11.1$)$ & $2 \times 10^{-33}$ & $-5.3(-7.3$ to -3.3$)$ & $3 \times 10^{-7}$ & 4 \\
\hline Smoking inhalation & $-17.2(-35.3$ to -0.8$)$ & 0.04 & $-39.3(-58.8$ to -19.9$)$ & $8 \times 10^{-5}$ & 5 \\
\hline$<8$ years in school & $-250.7(-274.3$ to -227.1$)$ & $2 \times 10^{-98}$ & $-25.9(-39.3$ to -12.4$)$ & $2 \times 10^{-4}$ & 6 \\
\hline C-reactive protein, mg/l & $-8.4(-10.1$ to -6.8$)$ & $4 \times 10^{-23}$ & $-2.8(-4.3$ to -1.2$)$ & $4 \times 10^{-4}$ & 7 \\
\hline Ever smoking & $-130.2(-149.1$ to -111.2$)$ & $1 \times 10^{-43}$ & Omitted* & - & - \\
\hline Exposure to dust or fumes & $-66.5(-92.5$ to -40.4$)$ & $2 \times 10^{-7}$ & Omitted* & - & - \\
\hline
\end{tabular}

unadjusted analyses, OR for the shortest versus the longest telomere quartiles was 2.06 (95\% CI 1.91 to 2.22) for GOLD criteria and 1.73 (1.60 to 1.88 ) for $\mathrm{FEV}_{1} / \mathrm{FVC}$ below LLN. The association was markedly attenuated when analyses were adjusted for age or multivariable adjusted for age, gender, body mass index, smoking status, smoking inhalation, cumulative smoking, occupational exposure to dust and fumes, years in school, C-reactive protein levels, grade of dyspnoea and study. In the multivariable models the OR for the shortest versus longest telomere quartiles was 1.15 (95\% CI 1.06 to 1.25$)$ for GOLD criteria and 1.19 (95\% CI 1.09 to 1.30 ) for $\mathrm{FEV}_{1} / \mathrm{FVC}$ below LLN. Trend tests across quartiles after multivariable adjustment yielded $\mathrm{p}$ values of 0.001 and $6 \times 10^{-5}$, respectively, for GOLD criteria and $\mathrm{FEV}_{1} / \mathrm{FVC}$ below LLN.
Per 1000 base pairs decrease in telomere length, the multivariable adjusted OR was 1.07 (95\% CI 1.03 to 1.10$)$ for GOLD criteria and 1.07 (1.03 to 1.11) for $\mathrm{FEV}_{1} / \mathrm{FVC}$ below LLN (figure 6). We stratified according to gender, age, smoking status and cumulative smoking in multivariable adjusted models and did not find evidence of an interaction between age, smoking status or cumulative smoking and decreasing telomere length on risk of COPD classified as GOLD or as $\mathrm{FEV}_{1} / \mathrm{FVC}$ below LLN. For GOLD we found a $\mathrm{p}$ value for test of interaction of 0.01 for men compared with women (required $\mathrm{p}$ value after Bonferroni correction $<0.05 / 4=0.013)$. However, the corresponding $\mathrm{p}$ value for $\mathrm{FEV}_{1} / \mathrm{FVC}$ below LLN was 0.75 .

The results from the two studies separately are shown in tables S2-S5 and figures S1-S4 in the online supplement. The

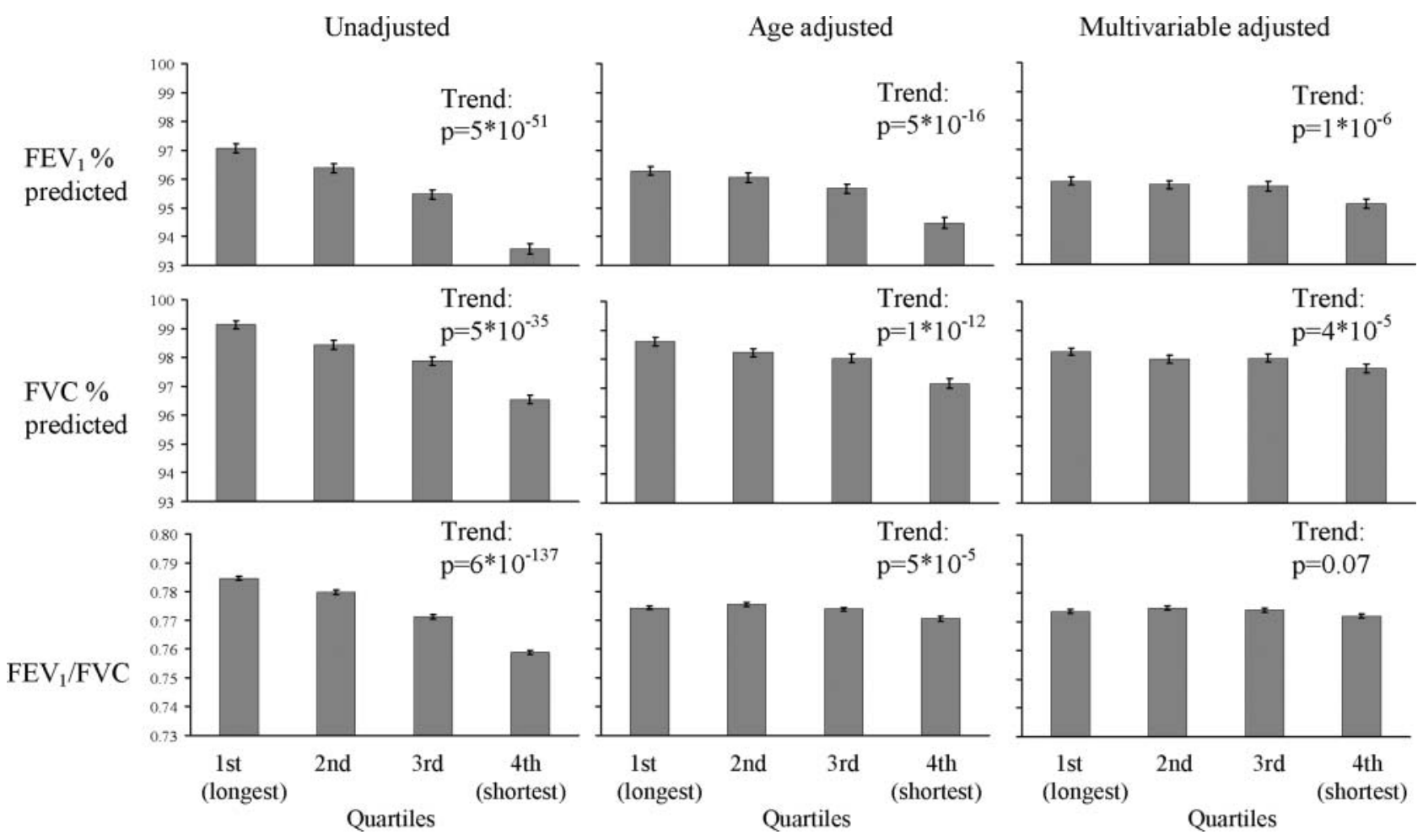

Figure 2 Forced expiratory volume in $1 \mathrm{~s}\left(\mathrm{FEV}_{1}\right) \%$ predicted, forced vital capacity (FVC) \% predicted and $\mathrm{FEV} / \mathrm{FVC}$ by quartiles of telomere length. Values are shown as mean and SE. Multivariable adjustments include age, gender, body mass index, smoking status, smoking inhalation, cumulative smoking in pack-years, occupational exposure to dust and fumes, years in school, plasma C-reactive protein levels, grade of dyspnoea and study. 


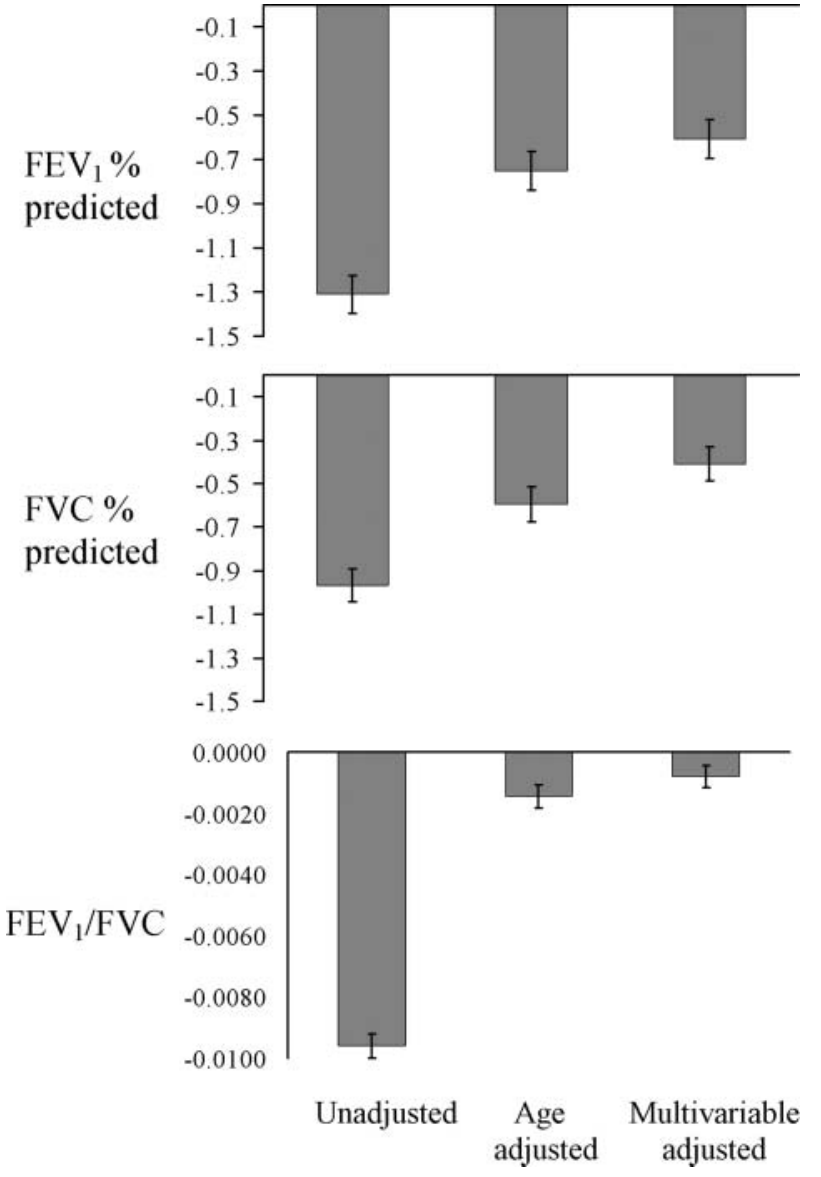

Figure 3 Changes in forced expiratory volume in $1 \mathrm{~s}\left(\mathrm{FEV}_{1}\right) \%$ predicted, forced vital capacity (FVC) \% predicted and $\mathrm{FEV}_{1} / \mathrm{FVC}$ for a 1000 base pair decrease in telomere length. Values are shown as mean and SE. Multivariable adjustments include age, gender, body mass index, smoking status, smoking inhalation, cumulative smoking in pack-years, occupational exposure to dust and fumes, years in school, plasma C-reactive protein levels, grade of dyspnoea and study. two studies showed similar results for all analyses. We repeated all analyses excluding individuals where a telomere length measurement had been obtained after a rerun of the blood sample. These sensitivity analyses did not change any of the main results or conclusions (data not shown).

\section{DISCUSSION}

The most important and novel finding from this observational study of a large sample of the Danish general population is that, although short telomere length is associated with reduced lung function and with an increased risk of COPD, these associations are attenuated markedly after age and multivariable adjustment. However, even after multivariable adjustment, a decrease in telomere length of 1000 base pairs was associated with a 1.07-fold risk of COPD by GOLD criteria as well as by $\mathrm{FEV}_{1} /$ FVC below LLN. Nevertheless, we cannot totally exclude that this remaining association could simply be a result of residual confounding. These results demonstrate an effect, albeit small, of an independent association between reduced telomere length and the risk of lung disease. It could be argued that telomere length is a marker of premature ageing and, as such, a mechanism by which other factors such as age, gender and smoking act. Therefore, telomere length could remain of interest even if not independently associated with lung function or risk of COPD.

The biological mechanism behind the finding of an association between decreased telomere length and decreased lung function and increased risk of COPD could be accelerated cell turnover in individuals with COPD, caused by inflammation and oxidative stress induced by inhalation of factors such as nitrogen dioxide, ozone, gasoline and diesel exhaust and tobacco smoke. Another hypothesis is that ageing and exposure to cigarette smoke entail lymphocyte senescence which increase susceptibility to infection and eventually lead to the chronic inflammation characteristic of COPD. ${ }^{21}$ COPD may also be associated with a genetic predisposition to shorter telomere length due to decreased telomerase activity, a theory supported by findings of shorter telomere length and lower telomerase activity in cultured pulmonary cells from patients with COPD

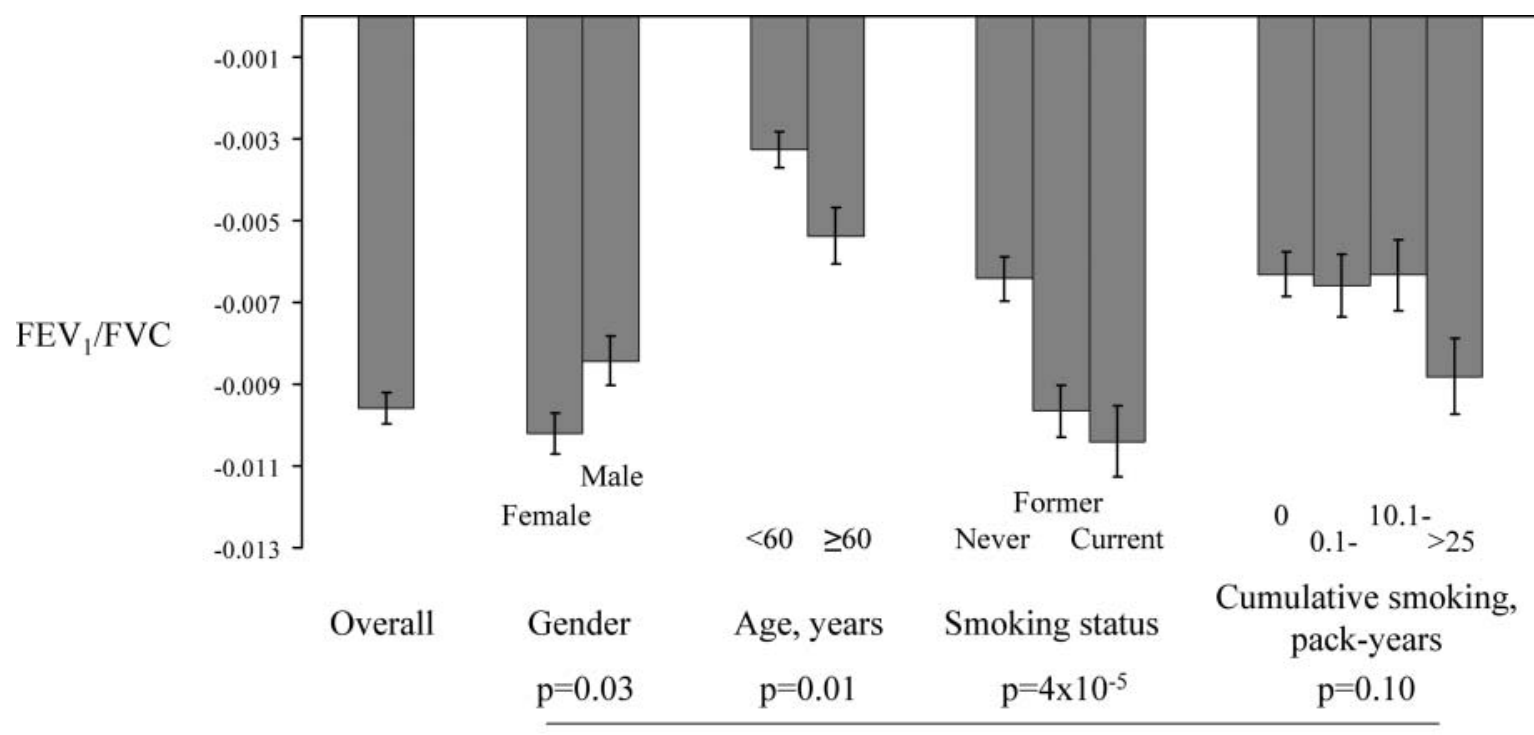

Test of interaction

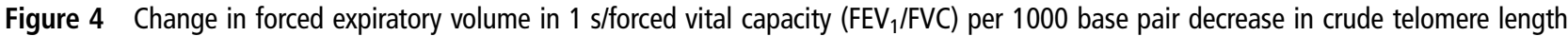
according to gender, age, smoking status and cumulative smoking stratifications. Unadjusted values are shown as mean and SE. 


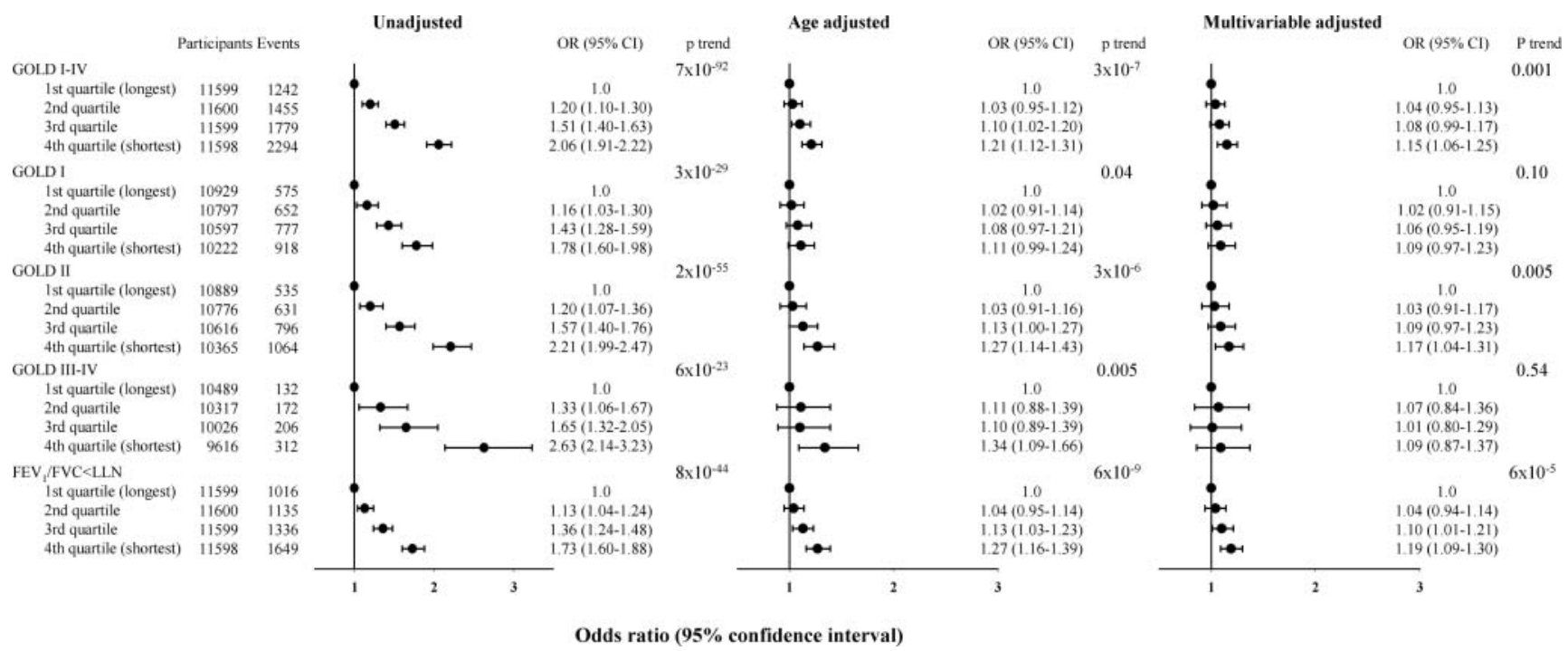

Figure 5 Logistic regression analysis of risk of chronic obstructive pulmonary disease (COPD) according to telomere length in quartiles. Multivariable adjustments include age, gender, body mass index, smoking status, smoking inhalation, cumulative smoking in pack-years, occupational exposure to dust and fumes, years in school, plasma C-reactive protein levels, grade of dyspnoea and study. COPD was defined either by the Global initiative for chronic Obstructive Lung Disease (GOLD) criteria or by forced expiratory volume in 1 s/forced vital capacity ratio $\left(\mathrm{FEV}_{1} / \mathrm{FVC}\right)$ below lower limit of normal.

compared with control subjects. ${ }^{22}$ Also, in a recent study Alder et $a l^{24}$ examined telomerase null mice with short telomeres and found that short telomere length could be a sign of genetic disposition to emphysema by increasing susceptibility to cigarette smoke-induced lung damage. Taken together, it is biologically plausible that short telomere length is associated with reduced lung function and an increased risk of COPD; however, the precise mechanism is unknown.

Our results are supported by previous findings of an association between telomere length and lung function and risk of COPD. ${ }^{25-29}$ Published studies have mainly been case-control studies of 26-283 COPD cases and 20-155 control subjects. In our cohort of 6770 individuals with COPD we found a 1.15 -fold increased risk of COPD for individuals with the shortest telomere lengths compared with those with the longest telomere lengths. To contrast this with previous findings, Savale et $a l^{25}$ compared 50 COPD patients with 50 matched control subjects and found a 28 -fold increased risk of COPD for individuals with short telomere length compared with individuals with long telomere length. Two other small case-control studies were unable to show an association between telomere length and lung function but found an association between smoking exposure and telomere length. ${ }^{26}{ }^{27}$ Mui et $a l^{28}$ also demonstrated an association between telomere length and lung function, but this study included COPD cases only. Overall, the results of previous studies support the notion that short telomere length is associated with decreased lung function and hence the risk of COPD. The present study of a very large

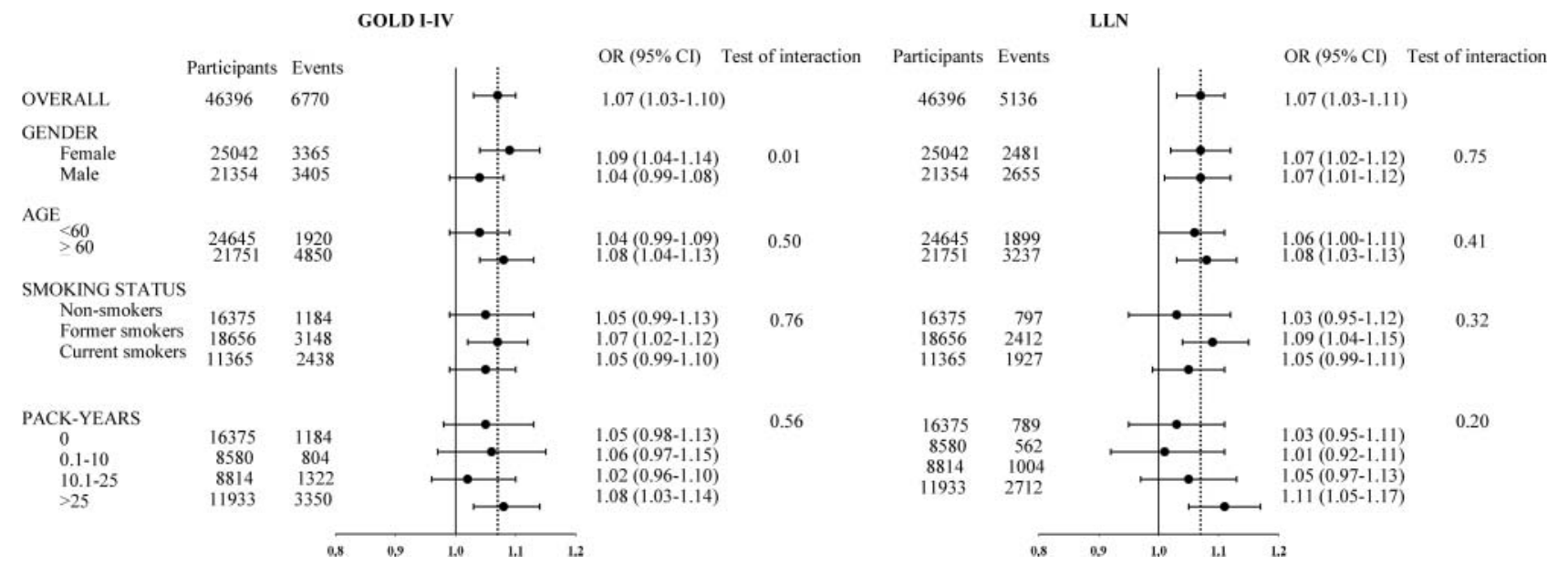

Figure 6 Risk of being classified as chronic obstructive pulmonary disease (COPD) by the Global initiative for chronic Obstructive Lung Disease (GOLD) criteria or by forced expiratory volume in 1 s/forced vital capacity (FEV $1 / F V C$ ) below lower limit of normal (LLN) per 1000 base pair decrease in telomere length. Multivariable adjustments include age, gender, body mass index, smoking status, smoking inhalation, cumulative smoking in pack-years, occupational exposure to dust and fumes, years in school, plasma C-reactive protein levels, grade of dyspnoea and study. COPD was defined either by the GOLD criteria or by $\mathrm{FEV}_{1} / \mathrm{FVC}$ ratio below LLN. 
sample from a general population suggests that the association is more moderate than previously reported in smaller case-control studies.

A strength of our study is the large cohort with telomere length measurement and spirometry data from 46396 individuals from the Danish general population. Also, importantly, we used two different classifications of COPD, examining 6770 individuals classified as COPD by GOLD criteria and 5136 by $\mathrm{FEV}_{1} / \mathrm{FVC}$ below LLN, and found similar results for the two COPD classifications. In addition, we combined data from two independent studies to obtain additional power, but the fact that these studies showed comparable results individually for the association between short telomeres and decreased lung function and increased risk of COPD adds additional credibility to the present study. Finally, the very strong association between decreasing telomere length and increasing age demonstrates the quality of our telomere length measurement.

One limitation of our study is that we cannot exclude the presence of residual confounding, despite the fact that we have included potential confounders such as age, gender, body mass index, smoking status, smoking inhalation, cumulative smoking, occupational exposure to dust and fumes, educational level, C-reactive protein levels and dyspnoea. Another limitation is that we did not have information on 6-min walking distance and therefore were not able to adjust for the entire Body mass index, airway Obstruction, Dyspnoea, and Exercise capacity (BODE) Index. ${ }^{30}$ Nevertheless, we did adjust for the three most important factors-namely, lung function, body mass index and grade of dyspnoea. The finding that the association was only moderate could also be explained by an underrepresentation of the most severe cases of COPD in our population, which may have underestimated the association between short telomere length and risk of COPD in this present study. Finally, as we studied white subjects only, our results may not necessarily apply to other races.

In conclusion, short telomere length is associated with decreased lung function and with increased risk of COPD, according to the GOLD classification as well as according to $\mathrm{FEV}_{1} / \mathrm{FVC}$ below LLN. However, the association seems to be only moderate and our results stress the importance of adjusting for age and other confounders when examining the association between COPD and short telomeres. Whether telomere shortening per se increases risk of COPD is still an unresolved question and should be examined in a study designed to evaluate the causal direction of the association.

Acknowledgements We thank laboratory technician Anja Jochumsen for assisting with the telomere measurements. The authors are indebted to the staff and participants in the Copenhagen General Population Study and the Copenhagen City Heart Study.

Contributors LR, SEB and BGN initiated the study. LR performed statistical analyses supervised by SEB, JV and BGN. All authors analysed and interpreted the results. BGN, MW and SEB collected data. LR drafted the manuscript which was scrutinised by the other authors, all of whom accepted the final submitted manuscript.

Funding This study was financially supported by Chief Physician Johan Boserup and Lise Boserup's Foundation, the Copenhagen County Foundation, Herlev Hospital, Copenhagen University Hospital and the Danish Heart Foundation.

Competing interests None.

Patient consent Written informed consent was obtained from all participants.

Ethics approval Ethical approval was obtained from Herlev Hospital and Danish ethics committees (H-KF01-144/01 and KF100.2039/91).

Provenance and peer review Not commissioned; internally peer reviewed.

\section{REFERENCES}

1 Rahman I. The role of oxidative stress in the pathogenesis of COPD: implications for therapy. Treat Respir Med 2005;4:175-200.

2 Donate LE, Blasco MA. Telomeres in cancer and ageing. Philos Trans $R$ Soc Lond $B$ Biol Sci 2011;366:76-84.

3 Stewart SA, Weinberg RA. Telomeres: cancer to human aging. Annu Rev Cell Dev Biol 2006;22:531-57.

4 Shammas MA. Telomeres, lifestyle, cancer, and aging. Curr Opin Clin Nutr Metab Care 2011:14:28-34.

5 von Zglinicki T. Oxidative stress shortens telomeres. Trends Biochem Sci 2002;27:339-44.

6 Cherkas LF, Aviv A, Valdes AM, et al. The effects of social status on biological aging as measured by white-blood-cell telomere length. Aging Cell 2006;5:361-5.

7 Valdes AM, Andrew T, Gardner JP, et al. Obesity, cigarette smoking, and telomere length in women. Lancet 2005;366:662-4.

8 von Zglinicki T, Martin-Ruiz CM. Telomeres as biomarkers for ageing and age-related diseases. Curr Mol Med 2005;5:197-203.

9 Cassidy A, De Vivo I, Liu Y, et al. Associations between diet, lifestyle factors, and telomere length in women. Am J Clin Nutr 2010;91:1273-80.

10 Vestbo J, Hurd SS, Agusti AG, et al. Global strategy for the diagnosis, management and prevention of chronic obstructive pulmonary disease, GOLD executive summary. Am J Respir Crit Care Med Published Online First: 9 Aug 2012. doi: 10.1164/ rccm.201204-0596PP

11 Swanney MP, Ruppel G, Enright PL, et al. Using the lower limit of normal for the $\mathrm{FEV}_{1} / \mathrm{FVC}$ ratio reduces the misclassification of airway obstruction. Thorax 2008:63:1046-51.

12 Baekvad-Hansen M, Dahl M, Tybjaerg-Hansen A, et al. Surfactant protein-B 121 ins2 heterozygosity, reduced pulmonary function, and chronic obstructive pulmonary disease in smokers. Am J Respir Crit Care Med 2010;181:17-20.

13 Dahl M, Bowler RP, Juul K, et al. Superoxide dismutase 3 polymorphism associated with reduced lung function in two large populations. Am J Respir Crit Care Med 2008;178:906-12.

14 Nordestgaard BG, Palmer TM, Benn M, et al. The effect of elevated body mass index on ischemic heart disease risk: causal estimates from a Mendelian randomisation approach. PLoS Med 2012;9:e1001212.

15 Dahl $M$, Tybjaerg-Hansen $A$, Lange $P$, et al. Change in lung function and morbidity from chronic obstructive pulmonary disease in alpha1-antitrypsin MZ heterozygotes: a longitudinal study of the general population. Ann Intern Med 2002;136:270-9.

16 Juul K, Tybjaerg-Hansen A, Mortensen J, et al. Factor V leiden homozygosity, dyspnea, and reduced pulmonary function. Arch Intern Med 2005;165:2032-6.

17 Kaur-Knudsen D, Nordestgaard BG, Bojesen SE. CHRNA3 genotype, nicotine dependence, lung function and disease in the general population. Eur Respir $J$ 2012:40:1538-44.

18 Cawthon RM. Telomere length measurement by a novel monochrome multiplex quantitative PCR method. Nucleic Acids Res 2009;37:e21.

19 Weischer M, Bojesen SE, Cawthon RM, et al. Short telomere length, myocardial infarction, ischemic heart disease, and early death. Arterioscler Thromb Vasc Biol 2012;32:822-9

20 Bestall JC, Paul EA, Garrod R, et al. Usefulness of the Medical Research Counci (MRC) dyspnoea scale as a measure of disability in patients with chronic obstructive pulmonary disease. Thorax 1999;54:581-6.

21 Aoshiba K, Nagai A. Senescence hypothesis for the pathogenetic mechanism of chronic obstructive pulmonary disease. Proc Am Thorac Soc 2009;6:596-601.

22 Amsellem V, Gary-Bobo G, Marcos E, et al. Telomere dysfunction causes sustained inflammation in chronic obstructive pulmonary disease. Am J Respir Crit Care Med 2011;184:1358-66.

23 Tsuji T, Aoshiba K, Nagai A. Alveolar cell senescence in patients with pulmonary emphysema. Am J Respir Crit Care Med 2006;174:886-93.

24 Alder JK, Guo N, Kembou F, et al. Telomere length is a determinant of emphysema susceptibility. Am J Respir Crit Care Med 2011;184:904-12.

25 Savale L, Chaouat A, Bastuji-Garin S, et al. Shortened telomeres in circulating leukocytes of patients with chronic obstructive pulmonary disease. Am J Respir Crit Care Med 2009;179:566-71.

26 Houben JM, Mercken EM, Ketelslegers HB, et al. Telomere shortening in chronic obstructive pulmonary disease. Respir Med 2009;103:230-6.

27 Morla M, Busquets X, Pons J, et al. Telomere shortening in smokers with and without COPD. Eur Respir J 2006;27:525-8.

28 Mui TS, Man JM, McElhaney JE, et al. Telomere length and chronic obstructive pulmonary disease: evidence of accelerated aging. J Am Geriatr Soc 2009;57:2372-4.

29 Lee J, Sandford AJ, Connett JE, et al. The relationship between telomere length and mortality in Chronic Obstructive Pulmonary Disease (COPD). PLoS One 2012;7: e35567.

30 Celli BR, Cote CG, Marin JM, et al. The body-mass index, airflow obstruction, dyspnea, and exercise capacity index in chronic obstructive pulmonary disease. $N$ Engl J Med 2004;350:1005-12. 